\title{
Anchor Placement in TOA based Wireless Localization Networks via Convex Relaxation
}

\author{
Cuneyd Ozturk and Sinan Gezici \\ Department of Electrical and Electronics Engineering, Bilkent University, Ankara, Turkey \\ \{cuneyd,gezici\}@ee.bilkent.edu.tr
}

\begin{abstract}
A wireless source localization network consisting of synchronized target and anchor nodes is considered. An anchor placement problem is formulated to minimize the Cramér-Rao lower bound (CRLB) on estimation of target node positions by anchor nodes. First, it is shown that the anchor placement problem can be approximated as a minimization problem of the ratio of two supermodular functions. Due to the lack of a polynomial time algorithm for such problems, an anchor selection problem is proposed to solve the anchor placement problem. Via relaxation of integer constraints, the anchor selection problem is approximated by a convex optimization problem, which is used to propose two algorithms for anchor selection. Furthermore, extensions to quasi-synchronous wireless localization networks are discussed. To examine the performance of the proposed algorithms, various simulation results are presented.
\end{abstract}

Index Terms-Localization, anchor placement, estimation, CRLB.

\section{INTRODUCTION}

In wireless localization networks, location estimation is performed via signal exchanges between target and anchor nodes in order to determine positions of target nodes [1], [2]. There exist two main types of wireless localization networks; namely, self localization and source (network centric) localization networks. Target nodes estimate their positions from signals transmitted from anchor nodes in self localization networks. On the other hand, anchor nodes estimate positions of target nodes from signals emitted by target nodes in source localization networks.

For both self localization and source localization networks, the main goal is to maintain high localization accuracy [1]. Determining where to place anchor nodes and how to allocate power among them are crucial issues to address for achieving high localization accuracy. There exist a vast amount of studies on these issues such as [3]-[12]. For example, in [6], anchor positions are assumed to be known, and optimization problems for optimal power allocation among anchor nodes, anchor selection, and anchor deployment are proposed to minimize the estimation error for target position considering a timeof-arrival (TOA) based approach. The problem of anchor placement is also investigated in [11], and a near-optimal node placement algorithm is proposed. In addition to anchor and target nodes, assisting nodes are placed in the network to improve localization accuracy, and an upper bound on the gap between the near-optimal node placement algorithm and the optimal placement strategy is provided [11]. Apart from the TOA based approach, there also exist some studies that consider the optimal anchor placement problem for time-differenceof-arrival (TDOA) and angle-of-arrival (AOA) based wireless localization networks [7], [12].
In this work, we consider a wireless source localization network consisting of anchor and target nodes, and propose an anchor selection problem to solve the anchor placement problem. The main contributions of this manuscript can be summarized as follows:

- We formulate the anchor placement problem for TOA based source localization networks by considering the Cramér-Rao lower bound (CRLB) as a performance metric, and we discretize the problem due to its nonconvexity.

- After discretization, we prove the equivalence of this problem to the problem of minimizing the ratio of two supermodular functions. Due to the lack of a polynomial time algorithm for such problems [13], we express the resulting problem as an anchor selection problem.

- Even though the problem of anchor selection is considered in [6], its convexity properties are not investigated and specific algorithms are not proposed to solve it. We prove that the objective function of this problem is convex with respect to the anchor selection vector. After relaxing the integer constraints, we come up with a convex optimization problem, which is related to the solution of the anchor selection problem.

- We propose two algorithms to solve the proposed anchor selection problem and present simulation results for both two and three dimensional wireless localization networks.

- We also show that this analysis can easily be extended to TDOA based wireless localization networks.

\section{SYSTEM MODEL}

Consider a two-dimensional wireless source localization network in which a target (source) node sends signals to anchor (reference) nodes for estimation of the target node location by anchor nodes. It is known that the target node is located at $\mathbf{x}_{i} \in \mathbb{R}^{2}$ with probability $w_{i} \geq 0$ for $i=1,2, \ldots, N_{T}$, where $N_{T}$ is the number of possible target locations and $\sum_{i=1}^{N_{T}} w_{i}=1$. Anchor nodes are located at $\left\{\mathbf{y}_{j}\right\}_{j=1}^{N_{A}} \subset \mathbb{R}^{2}$, where $N_{A}$ is the number of anchor nodes in the network. $\phi_{i j}$ is the angle between $j$ th anchor node and $i$ th target node, i.e., $\phi_{i j}=\arctan \frac{x_{i 2}-y_{j 2}}{x_{i 1}-y_{j 1}}$, where $\mathbf{x}_{i}=\left[x_{i 1} x_{i 2}\right]^{\top}$ and $\mathbf{y}_{j}=\left[\begin{array}{ll}y_{j 1} & y_{j 2}\end{array}\right]^{\top}$.

In the following sections, we provide the signal model and formulate the corresponding anchor placement problems for TOA and TDOA based wireless localization networks. 


\section{TOA BASED ANChOR Placement Problem}

\section{A. Signal Model}

Consider a scenario in which the clocks of the anchor and target nodes are perfectly synchronized. For the signal emitted from the target node located at $\mathbf{x}_{i}$, the signal received at the anchor node at location $\mathbf{y}_{j}$ is given by

$$
r_{i j}(t)=\sum_{l=1}^{L_{i j}} \alpha_{i j}^{(l)} s_{i}\left(t-\tau_{i j}^{(l)}\right)+\eta_{i j}(t)
$$

where $s_{i}(t)$ is a known signal transmitted from the $i$ th target node, $L_{i j}$ is the number of multipath components between the $j$ th anchor node and the $i$ th target node, $\alpha_{i j}^{(l)}$, and $\tau_{i j}^{(l)}$ denote, respectively, the amplitude and the delay of the $l$ th multipath component between the $j$ th anchor node and the $i$ th target node, and $\eta_{i j}(t)$ is the measurement noise modeled as zeromean white Gaussian noise with a power spectral density level of $N_{0} / 2$. The delays of the paths are given by

$$
\tau_{i j}^{(l)}=\frac{\left\|\mathbf{y}_{j}-\mathbf{x}_{i}\right\|+b_{i j}^{(l)}}{c}
$$

where $c$ is the speed of the light and $b_{i j}^{(l)}$ is the range-bias term $\left(b_{i j}^{(l)}=0\right.$ for line-of-sight (LOS) propagation and $b_{i j}^{(l)}>0$ for non-line-of-sight (NLOS) propagation).

\section{B. Problem Formulation}

In the considered wireless localization network, the aim is to determine the best $N_{A}$ locations to place the anchor nodes in order to estimate the location of the target node as accurately as possible in the mean-square sense. In other words, $\mathbf{y}_{j}$ 's are the optimization variables in the anchor placement problem. In order to emphasize that, we introduce a matrix $\mathbf{Y}$ as follows: $\mathbf{Y} \triangleq\left[\begin{array}{llll}\mathbf{y}_{1} & \mathbf{y}_{2} & \ldots & \mathbf{y}_{N_{A}}\end{array}\right]$. It is assumed that each anchor node can be placed in some bounded region $\mathcal{R} \subset \mathbb{R}^{2}$, that is, $\mathbf{y}_{j} \in \mathcal{R}$ for $j=1, \ldots, N_{A}$.

After defining the anchor positions as optimization variables, the number, amplitudes and delays of the multipath components, and the angles between the anchor and target nodes become functions of the anchor positions. In order to emphasize the dependence on $\mathbf{Y}$, we replace $L_{i j}, \alpha_{i j}^{(l)}$, $\tau_{i j}^{(l)}$, and $\phi_{i j}$ with $L_{i}\left(\mathbf{y}_{j}\right), \alpha_{i}^{(l)}\left(\mathbf{y}_{j}\right), \tau_{i}^{(l)}\left(\mathbf{y}_{j}\right)$, and $\phi_{i}\left(\mathbf{y}_{j}\right)$, respectively, in the remainder of the manuscript.

Via similar steps to those in [14], the equivalent Fisher information matrix (EFIM), $\mathbf{J}_{i}^{\mathrm{TOA}}(\mathbf{Y})$, corresponding to the target location $\mathbf{x}_{i}$ can be expressed as a function of the anchor locations $\left\{\mathbf{y}_{j}\right\}_{j=1}^{N_{A}}$ as

$$
\begin{aligned}
& \mathbf{J}_{i}^{\mathrm{TOA}}(\mathbf{Y})=\sum_{j \in \mathcal{A}_{i}^{L}(\mathbf{Y})} \lambda_{i}\left(\mathbf{y}_{j}\right) \boldsymbol{\varphi}_{i}\left(\mathbf{y}_{j}\right) \boldsymbol{\varphi}_{i}\left(\mathbf{y}_{j}\right)^{\top} \\
& \lambda_{i}\left(\mathbf{y}_{j}\right) \triangleq \frac{8 \pi^{2} \beta_{i}^{2}}{c^{2}}\left(1-\xi_{i}\left(\mathbf{y}_{j}\right)\right) \frac{\left|\alpha_{i}^{(1)}\left(\mathbf{y}_{j}\right)\right|^{2} \int_{-\infty}^{\infty}\left|S_{i}(f)\right|^{2} d f}{N_{0}} \\
& \beta_{i}^{2} \triangleq \frac{\int_{-\infty}^{\infty} f^{2}\left|S_{i}(f)\right|^{2} d f}{\int_{-\infty}^{\infty}\left|S_{i}(f)\right|^{2} d f}, \boldsymbol{\varphi}_{i}\left(\mathbf{y}_{j}\right) \triangleq\left[\cos \left(\phi_{i}\left(\mathbf{y}_{j}\right)\right) \sin \left(\phi_{i}\left(\mathbf{y}_{j}\right)\right)\right]^{\top}
\end{aligned}
$$

where $\mathcal{A}_{i}^{L}(\mathbf{Y})$ is the set of indices of anchor nodes that are in LOS with the target node at location $\mathbf{x}_{i}$ for the anchor positions given by matrix $\mathbf{Y}, S_{i}(f)$ is the Fourier transform of $s_{i}(t)$, and $\xi_{i}\left(\mathbf{y}_{j}\right)$ is the path-overlap coefficient for the anchor location $\mathbf{y}_{j}$ and the target location $\mathbf{x}_{i}$ satisfying $0 \leq \xi_{i}\left(\mathbf{y}_{j}\right) \leq$ 1 [14].

It is known that for any unbiased estimator $\hat{\mathbf{x}}_{i}$ of $\mathbf{x}_{i}$, the error vector satisfies [15]:

$$
\mathbb{E}\left\{\left\|\hat{\mathbf{x}}_{i}-\mathbf{x}_{i}\right\|^{2}\right\} \geq \operatorname{tr}\left\{\mathbf{J}_{i}^{\mathrm{TOA}}(\mathbf{Y})^{-1}\right\}
$$

where the expression on the right-hand-side corresponds to the CRLB. Then, by considering the CRLB as a performance metric, the proposed anchor placement problem can be formulated as

$$
\begin{array}{ll}
\min _{\mathbf{Y}} & \sum_{i=1}^{N_{T}} w_{i} \operatorname{tr}\left\{\mathbf{J}_{i}^{\mathrm{TOA}}(\mathbf{Y})^{-1}\right\} \\
\text { subject to } & \mathbf{y}_{j} \in \mathcal{R}, j=1,2, \ldots, N_{A} .
\end{array}
$$

\section{Theoretical Results and Algorithms}

From (3) and (5), $\operatorname{tr}\left\{\mathbf{J}_{i}^{\mathrm{TOA}}(\mathbf{Y})^{-1}\right\}$ can be expressed after some algebraic manipulations as

$$
\begin{aligned}
& \operatorname{tr}\left\{\mathbf{J}_{i}^{\mathrm{TOA}}(\mathbf{Y})^{-1}\right\}= \\
& \frac{2 \sum_{k \in \mathcal{A}_{i}^{L}(\mathbf{Y})} \lambda_{i}\left(\mathbf{y}_{k}\right)}{\sum_{k \in \mathcal{A}_{i}^{L}(\mathbf{Y})} \sum_{l \in \mathcal{A}_{i}^{L}(\mathbf{Y})} \lambda_{i}\left(\mathbf{y}_{k}\right) \lambda_{i}\left(\mathbf{y}_{l}\right) \sin ^{2}\left(\phi_{i}\left(\mathbf{y}_{k}\right)-\phi_{i}\left(\mathbf{y}_{l}\right)\right)}
\end{aligned}
$$

It is noted from (8) that the optimal anchor placement problem in (7) is quite challenging since the number of multipath components, the amplitudes, the delays, the angles between the anchor and target nodes, and the path-overlap coefficients become functions of the anchor locations (see (3)(5)). Moreover, this problem is not convex in general. For example, the set $\mathcal{R}$ may not necessarily be a convex set.

In order to solve the anchor placement problem, we first discretize set $\mathcal{R}$ in (7b). Let $\mathcal{R}$ be a finite subset of $\mathcal{R}$, and consider the problem in (7) for set $\widetilde{\mathcal{R}}$ by replacing the constraint in (7b) by $\mathbf{y}_{j} \in \widetilde{\mathcal{R}}$ for $j=1, \ldots, N_{A}$. Since the objective function in (7a) is continuous with respect to the anchor locations, the optimal value of the discretized version of (7) gets closer to the optimal value of (7) as the number of elements in $\widetilde{\mathcal{R}}$ increases. Therefore, it is possible to achieve an accurate approximation to the original problem in (7) via discretization.

In the discretized version of (7), the aim is to identify a subset of $\widetilde{\mathcal{R}}$ with a cardinality of $N_{A}$. As $\widetilde{\mathcal{R}}$ is finite, we can represent its elements as $\mathcal{R}=\left(\mathbf{y}^{(k)}\right)_{k}$. Furthermore, we define $\lambda_{i}^{(k)} \triangleq \lambda_{i}\left(\mathbf{y}^{(k)}\right), \phi_{i}^{(k)} \triangleq \phi_{i}\left(\mathbf{y}^{(k)}\right)$, and $\boldsymbol{\varphi}_{i}^{(k)} \triangleq \boldsymbol{\varphi}_{i}\left(\mathbf{y}^{(k)}\right)$ for any $i$ and $k$. Moreover, let the set of indices of anchor nodes in $\widetilde{\mathcal{R}}$ which are in LOS with the target node at location $\mathbf{x}_{i}$ be denoted as $\tilde{\mathcal{A}}_{i}^{L}$ for any $i$. For any set $\mathcal{S}$, we define $\mathcal{S}_{i}^{L} \triangleq \mathcal{S} \cap \tilde{\mathcal{A}}_{i}^{L}$. Then, the discretized version of (7) is expressed as

$$
\begin{array}{ll}
\min _{\mathcal{S} \subset \mathbb{N}} & \sum_{i=1} w_{i} f_{i}^{\mathrm{TO}} \\
\text { subject to } & |\mathcal{S}|=N_{A}
\end{array}
$$


where $\mathbb{N}$ denotes the set of natural numbers and $f_{i}^{\mathrm{TOA}}(\mathcal{S})$ is given by

$$
f_{i}^{\mathrm{TOA}}(\mathcal{S}) \triangleq \frac{2 \sum_{k \in \mathcal{S}_{i}^{L}} \lambda_{i}^{(k)}}{\sum_{k \in \mathcal{S}_{i}^{L}} \sum_{l \in \mathcal{S}_{i}^{L}} \lambda_{i}^{(k)} \lambda_{i}^{(l)} \sin ^{2}\left(\phi_{i}^{(k)}-\phi_{i}^{(l)}\right)} .
$$

The following lemma proves that solving (9) is equivalent to minimizing the ratio of non-decreasing supermodular functions subject to a cardinality constraint.

Lemma 1: The objective function in (9) is a ratio of two non-decreasing supermodular functions.

Proof: Define $p_{i}^{\mathrm{TOA}}(\mathcal{S}) \triangleq 2 \sum_{k \in \mathcal{S}_{i}^{L}} \lambda_{i}^{(k)}$, and $q_{i}^{\mathrm{TOA}}(\mathcal{S}) \triangleq$ $\sum_{k \mathcal{S}_{i}^{L}} \sum_{l \in \mathcal{S}_{i}^{L}} \lambda_{i}^{(k)} \lambda_{i}^{(l)} \sin ^{2}\left(\phi_{i}^{(k)}-\phi_{i}^{(l)}\right)$.

If $\mathcal{S} \subset \mathcal{W}$, then $p_{i}^{\mathrm{TOA}}(\mathcal{S}) \leq p_{i}^{\mathrm{TOA}}(\mathcal{W})$ and $q_{i}^{\mathrm{TOA}}(\mathcal{S}) \leq$ $q_{i}^{\mathrm{TOA}}(\mathcal{W})$. In other words, both $p_{i}^{\mathrm{TOA}}(\cdot)$ and $q_{i}^{\mathrm{TOA}}(\cdot)$ are nondecreasing functions.

Take any $e \notin \mathcal{W}$. If $e \notin \tilde{\mathcal{A}}_{i}^{L}$, then it is clear that $p_{i}^{\mathrm{TOA}}(\mathcal{W} \cup\{e\})-p_{i}^{\mathrm{TOA}}(\mathcal{W})=p_{i}^{\mathrm{TOA}}(\mathcal{S} \cup\{e\})-p_{i}^{\mathrm{TOA}}(\mathcal{S})=0$, and $q_{i}^{\mathrm{TOA}}(\mathcal{W} \cup\{e\})-q_{i}^{\mathrm{TOA}}(\mathcal{W})=q_{i}^{\mathrm{TOA}}(\mathcal{S} \cup\{e\})-q_{i}^{\mathrm{TOA}}(\mathcal{S})=$ 0 . If $e \in \tilde{\mathcal{A}}_{i}^{L}$, it is observed that

$$
\begin{aligned}
p_{i}^{\mathrm{TOA}}(\mathcal{W} \cup\{e\})-p_{i}^{\mathrm{TOA}}(\mathcal{W}) & =p_{i}^{\mathrm{TOA}}(\mathcal{S} \cup\{e\})-p_{i}^{\mathrm{TOA}}(\mathcal{S}) \\
q_{i}^{\mathrm{TOA}}(\mathcal{W} \cup\{e\})-q_{i}^{\mathrm{TOA}}(\mathcal{W}) & =2 \lambda_{i}^{(e)} \sum_{k \in \mathcal{W}} \lambda_{i}^{(k)} \sin ^{2}\left(\phi_{i}^{(k)}-\phi_{i}^{(e)}\right) \\
& \geq 2 \lambda_{i}^{(e)} \sum_{k \in \mathcal{S}} \lambda_{i}^{(k)} \sin ^{2}\left(\phi_{i}^{(k)}-\phi_{i}^{(e)}\right) \\
& =q_{i}^{\mathrm{TOA}}(\mathcal{S} \cup\{e\})-q_{i}^{\mathrm{TOA}}(\mathcal{S})
\end{aligned}
$$

Therefore, both $p_{i}^{\mathrm{TOA}}(\cdot)$ and $q_{i}^{\mathrm{TOA}}(\cdot)$ are non-decreasing supermodular functions [16]. Since the product of two nondecreasing, non-negative and supermodular functions is supermodular, it is concluded that $p_{i}^{\mathrm{TOA}}(\mathcal{S}) \prod_{j \neq i} q_{j}^{\mathrm{TOA}}(\mathcal{S})$ and $\prod_{j} q_{j}(\mathcal{S})$ are supermodular functions as $p_{i}^{\mathrm{TOA}}(\cdot)$ and $q_{i}^{\mathrm{TOA}}(\cdot)$ are supermodular, non-negative and non-decreasing. Based on the definitions at the beginning of the proof, the objective function in (9), which is specified via (10), can be expressed as

$$
\sum_{i=1}^{N_{T}} w_{i} f_{i}^{\mathrm{TOA}}(\mathcal{S})=\frac{\sum_{i=1}^{N_{T}} w_{i} p_{i}^{\mathrm{TOA}}(\mathcal{S}) \prod_{j \neq i} q_{j}^{\mathrm{TOA}}(\mathcal{S})}{\prod_{j} q_{j}^{\mathrm{TOA}}(\mathcal{S})} .
$$

Since supermodularity is preserved under non-negative weighted summation, $\sum_{i=1}^{N_{T}} w_{i} p_{i}^{\mathrm{TOA}}(\mathcal{S}) \prod_{j \neq i} q_{j}^{\mathrm{TOA}}(\mathcal{S})$ is also supermodular. Therefore, both the numerator and the denominator of the objective function are supermodular.

In [13], it is stated that whether optimizing the ratio of monotone supermodular functions admits any polynomial time algorithm with bounded approximations is an open problem. Therefore, instead of employing integer programming approaches, we aim to transform (9) into an anchor selection problem that can be solved via convex optimization tools. To this aim, we introduce a selection vector $\mathbf{z}=\left[\begin{array}{llll}z_{1} & z_{2} & \ldots & z_{\mid \widetilde{\mathcal{R}}}\end{array}\right]^{\top}$ as follows:

$$
z_{k}= \begin{cases}1, & \text { if an anchor node is placed at } \mathbf{y}^{(k)} \\ 0, & \text { otherwise }\end{cases}
$$

It is noted that (9) is equivalent to the following problem:

$$
\begin{array}{ll}
\min _{\mathbf{z} \in \mathbb{R}^{\mid \widetilde{\mathcal{R}}} \mid} & \sum_{i=1}^{N_{T}} w_{i} \tilde{f}_{i}^{\mathrm{TOA}}(\mathbf{z}) \\
\text { subject to } & \sum_{k=1}^{|\widetilde{\mathcal{R}}|} z_{k}=N_{A} \\
& z_{k} \in\{0,1\}, k=1,2, \ldots,|\widetilde{\mathcal{R}}|
\end{array}
$$

where $\tilde{f}_{i}^{\mathrm{TOA}}(\mathbf{z})$ is given by

$$
\tilde{f}_{i}^{\mathrm{TOA}}(\mathbf{z}) \triangleq \frac{2 \sum_{k \in \tilde{\mathcal{A}}_{i}^{L}} z_{k} \lambda_{i}^{(k)}}{\sum_{k \in \tilde{\mathcal{A}_{i}^{L}}} \sum_{l \in \tilde{\mathcal{A}}_{i}^{L}} z_{k} z_{l} \lambda_{i}^{(k)} \lambda_{i}^{(l)} \sin ^{2}\left(\phi_{i}^{(k)}-\phi_{i}^{(l)}\right)}
$$

The following proposition proves that the objective function in (13) is a convex function of the selection vector $\mathbf{z}$.

Proposition 1: The objective function in (13) is convex with respect to the selection vector $\mathbf{z}$.

Proof: It can be shown that $\tilde{f}_{i}^{\mathrm{TOA}}(\mathbf{z})$ in (13) is equal to $\operatorname{tr}\left\{\tilde{\mathbf{J}}_{i}^{\mathrm{TOA}}(\mathbf{z})^{-1}\right\}$, where

$$
\tilde{\mathbf{J}}_{i}^{\mathrm{TOA}}(\mathbf{z}) \triangleq \sum_{k \in \tilde{\mathcal{A}}_{i}^{L}} z_{k} \lambda_{i}^{(k)} \boldsymbol{\varphi}_{i}^{(k)}\left(\boldsymbol{\varphi}_{i}^{(k)}\right)^{\top} .
$$

First, it is clear that $\tilde{\mathbf{J}}_{i}^{\mathrm{TOA}}(\mathbf{z})$ is a linear function of $\mathbf{z}$. Moreover, it is known that $\operatorname{tr}\left\{\mathbf{A}^{-1}\right\}$ is a non-increasing convex function of the positive semi-definite matrix A [17]. Due to the concavity of $\tilde{\mathbf{J}}_{i}^{\mathrm{TOA}}(\mathbf{z})$, we can argue, based on the chain rule, that $\tilde{f}_{i}^{\mathrm{TOA}}(\mathbf{z})$ is convex with respect to $\mathbf{z}$ for any $i$. As $w_{i}$ 's in (13a) are non-negative, we have the desired conclusion about the convexity of the objective function in (13).

Since the objective function is convex with respect to $\mathbf{z}$, by relaxing the integer constraints in (13c), we obtain a convex optimization problem as follows:

$$
\begin{array}{ll}
\min _{\mathbf{z} \in \mathbb{R}^{|\mathcal{S}|}} & \sum_{i=1}^{N_{T}} w_{i} \tilde{f}_{i}^{\mathrm{TOA}}(\mathbf{z}) \\
\text { subject to } & \sum_{k=1}^{|\widetilde{\mathcal{R}}|} z_{k}=N_{A} \\
& 0 \leq z_{k} \leq 1, k=1,2, \ldots,|\mathcal{S}|
\end{array}
$$

To summarize the main approach, we first formulate the anchor placement problem as a continuous and non-convex optimization problem as in (7). Then, we take finitely many points from the region $\mathcal{R}$ to discretize the problem as in (9). Next, we prove that this problem is equivalent to minimizing the ratio of two supermodular functions, for which there exists no polynomial time algorithms with bounded approximations in the literature. Therefore, we express this problem as an anchor selection problem as in (13), and by relaxing the integer constraints, it is proven that a convex formulation can be achieved as in (16). Thus, we propose the anchor selection problem in (16) as a convex approximation of the anchor placement problem in (7). After solving (16) via convex optimization tools (such as CVX [18]), we propose the following 
two algorithms to obtain a solution of the original problem in (7). First, we can simply set the largest $N_{A}$ components of the solution of (16) to one, and the others to zero (called largest$N_{A}$ algorithm). Second, starting from this solution, we can use the swap algorithm [19]. In this algorithm, for each run, one checks whether there is a decrease in the objective function by simply swapping one of the $N_{A}$ selected locations with one of the $|\widetilde{\mathcal{R}}|-N_{A}$ locations that are not selected. The overall procedure starting from $\mathcal{R}$ and $N_{A}$ is outlined in Algorithm 1 , where $\mathbf{z}_{\text {relaxed }}, \mathbf{z}_{\text {largest }}$ and $\mathbf{z}_{\text {swap }}$ denote the optimal selection vectors obtained from (16) (e.g., via $\mathrm{CVX}$ ), the largest- $N_{A}$ algorithm and the swap algorithm, respectively.

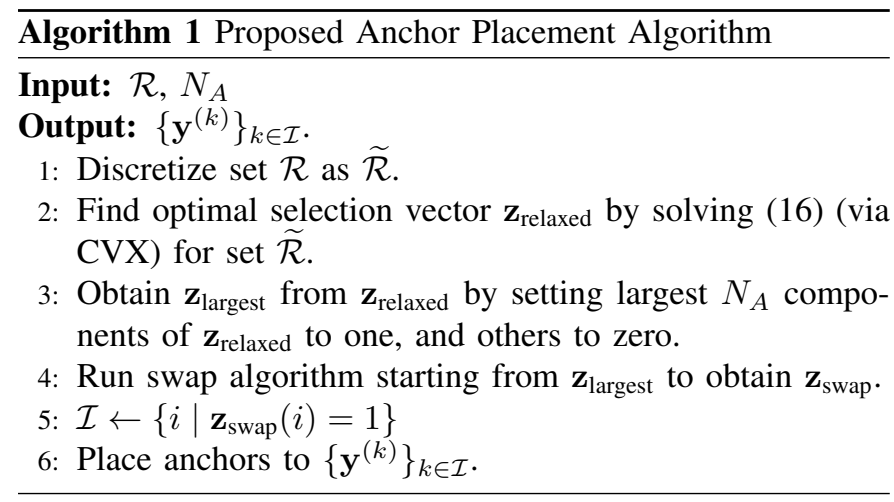

\section{Extension to Three-Dimensional Anchor Placement}

Similar to the analysis in [14], our results can easily be extended to three dimensional scenarios. In that case, $\boldsymbol{\varphi}_{i}\left(\mathbf{y}_{j}\right)$ in (5) becomes

$$
\begin{aligned}
& \boldsymbol{\varphi}_{i}\left(\mathbf{y}_{j}\right)=\left[\sin \theta_{i}\left(\mathbf{y}_{j}\right) \cos \phi_{i}\left(\mathbf{y}_{j}\right)\right. \\
& \left.\sin \theta_{i}\left(\mathbf{y}_{j}\right) \sin \phi_{i}\left(\mathbf{y}_{j}\right) \quad \cos \theta_{i}\left(\mathbf{y}_{j}\right)\right]^{\top}
\end{aligned}
$$

where $\theta_{i}\left(\mathbf{y}_{j}\right)$ and $\phi_{i}\left(\mathbf{y}_{j}\right)$ denote, respectively, the azimuth and polar angles in the direction from the target node at location $\mathbf{x}_{i}$ to the anchor node located at $\mathbf{y}_{j}$.

It is observed that after discretization and approximating the anchor placement problem as the anchor selection problem, Proposition 1 holds also for the three dimensional case as the FIM is a linear function of the selection vector $\mathbf{z}$ (cf. (15)). Hence, the proposed algorithms can be used for the three dimensional anchor placement problem, as well.

\section{TDOA Based Anchor Placement Problem}

In the TDOA based approach, the anchor nodes are synchronized among themselves but they are not synchronized with the target node. Therefore, there are additional unknown parameters $\left\{\Delta_{i}\right\}_{i=1}^{N_{T}}$, where $\Delta_{i}$ characterizes the time offset between the clocks of the target node located at $\mathbf{x}_{i}$ and the anchor nodes. Hence, (2) changes as follows:

$$
\tau_{i j}^{(l)}=\frac{1}{c}\left(\left\|\mathbf{y}_{j}-\mathbf{x}_{i}\right\|+b_{i j}^{(l)}+\Delta_{i}\right)
$$

As in the previous section, due to the difficulty of the continuous optimization problem (see (7)), we discretize the set $\mathcal{R}$ as $\widetilde{\mathcal{R}}$. After the discretization, via similar steps to those in
[19], one can show that the CRLB related to the estimation of the $i$ th target location, $\mathbf{x}_{i}$, by anchor nodes is given by

$$
f_{i}^{\mathrm{TDOA}}(\mathcal{S}) \triangleq \frac{3 \sum_{k \in \mathcal{S}_{i}^{L}} \sum_{l \in \mathcal{S}_{i}^{L}} \lambda_{i}^{(k)} \lambda_{i}^{(l)} a_{i}^{(k, l)}}{4 \sum_{k \in \mathcal{S}_{i}^{L}} \sum_{l \in \mathcal{S}_{i}^{L}} \sum_{m \in \mathcal{S}_{i}^{L}} \lambda_{i}^{(k)} \lambda_{i}^{(l)} \lambda_{i}^{(m)} \tilde{a}_{i}^{(k, l, m)}},
$$

$$
a_{i}^{(k, l)} \triangleq \sin ^{2}\left(\frac{\phi_{i}^{(k)}-\phi_{i}^{(l)}}{2}\right), \tilde{a}_{i}^{(k, l, m)} \triangleq a_{i}^{(k, l)} a_{i}^{(l, m)} a_{i}^{(m, k)} .
$$

Similar to Lemma 1, it can be proven that the objective function $\sum_{i=1}^{N_{T}} w_{i} f_{i}^{\mathrm{TDOA}}(\mathcal{S})$ can be written as a ratio of two non-decreasing supermodular functions. In addition, after relaxation, from Proposition 2 in [19], the anchor selection problem for the TDOA based approach can be shown to be convex, as well. Hence, the algorithms proposed for the TOA based approach in the previous section can also be used for TDOA based wireless localization networks.

Remark 1: Throughout the manuscript, a source localization network is considered. However, due to the similarity of the expressions for the FIMs, all the preceding analyses and the proposed algorithms are also valid for self localization networks (for both TOA and TDOA approaches).

Remark 2: In this manuscript, we rely on the perfect knowledge of $\lambda_{i}^{(k)}$,s. In fact, due to the monotonic behavior of the CRLB with respect to $\lambda_{i}^{(k)}$ 's, it is straightforward to extend the analyses if we have some uncertainty region for each $\lambda_{i}^{(k)}$ 's as in [19].

\section{Simulation Results and Conclusions}

In this section, numerical examples are presented to evaluate the proposed approaches in Section III. Examples are provided for two different setups in which two and three dimensional source localization networks are considered. In both setups, we evaluate the performance of two algorithms, namely, the largest- $N_{A}$ and the swap algorithms. We also compare their performance with the lower bound provided by the solution of the relaxed problem in (16).

\section{A. Example of Two Dimensional Wireless Network}

Consider a wireless source localization network as shown in Fig. 1 (all locations are in meters). In particular, the anchor nodes are placed in the region $\mathcal{R}=\mathcal{R}_{1} \cup \mathcal{R}_{2}$, where $\mathcal{R}_{1}=$ $[0,3] \times([0,3] \cup[8,11])$ and $\mathcal{R}_{2}=[8,11] \times([0,3] \cup[8,11])$. We provide two different discretizations of $\mathcal{R}$ by taking 196 and 676 points from $\mathcal{R}$. (We take points from $\mathcal{R}$ for each 0.5 and 0.25 meters along both $x$ and $y$ directions for the first and second setups, respectively.) Moreover, the number of possible locations for the target node, $N_{T}$, is equal to 129 as illustrated in Fig. 1.

In the simulations, the same parameters as in [19], [20] are used. In particular, $\alpha_{i}^{(l)}\left(\mathbf{y}_{j}\right)$ and $\xi_{i}\left(\mathbf{y}_{j}\right)$ are modeled as $\alpha_{i}^{(l)}\left(\mathbf{y}_{j}\right)=\left\|\mathbf{x}_{i}-\mathbf{y}_{j}\right\|^{-2}$ and $\xi_{i}\left(\mathbf{y}_{j}\right)=0$. Hence, $\lambda_{i}^{(k)}$ is expressed as $\lambda_{i}^{(k)}=8 \pi \beta_{i}^{2} E_{i} /\left(c^{2}\left\|\mathbf{x}_{i}-\mathbf{y}^{(k)}\right\|^{2} N_{0}\right)$. Also, the signal parameters are selected such that $\lambda_{i}^{(k)}=$ $1 /\left(\left\|\mathbf{x}_{i}-\mathbf{y}^{(k)}\right\|^{2} N_{0}\right)$ as in [20]. Also we set $w_{i}=1 / N_{T}$ for $i=1, \ldots, N_{T}$. The average CRLBs are presented in terms of meters, i.e., we take the square roots of the objectives. 


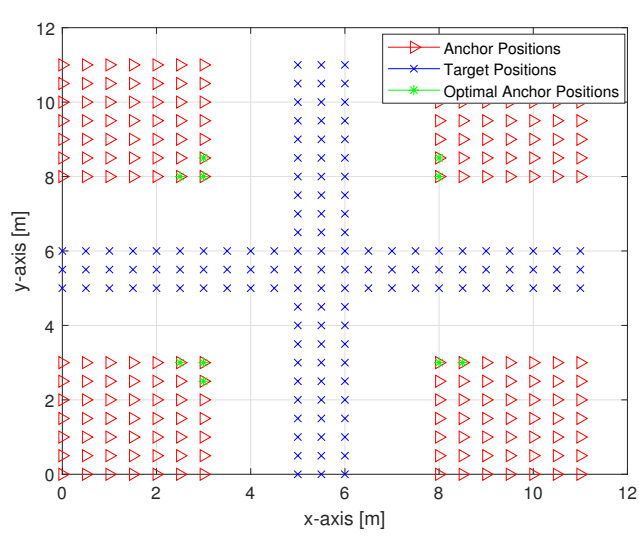

(a) $|\widetilde{\mathcal{R}}|=196$

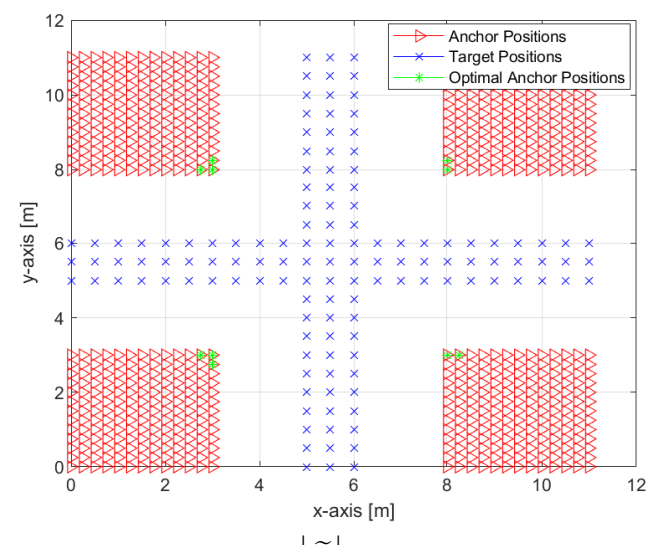

(b) $|\widetilde{\mathcal{R}}|=676$

Fig. 1: Illustration of possible locations of target and anchor nodes, together with optimal anchor locations (obtained from the swap algorithm) when $N_{A}=10$ and $N_{0}=1$ for two different cases: (a) $|\widetilde{\mathcal{R}}|=196$ and (b) $|\widetilde{\mathcal{R}}|=676$.

Fig. 1 shows the cases of $|\widetilde{\mathcal{R}}|=196$ and $|\widetilde{\mathcal{R}}|=676$, together with the optimal anchor locations obtained from the swap algorithm when $N_{A}=10$ and $N_{0}=1$. It is noted that even though the number of points in $\widetilde{\mathcal{R}}$ increases, the optimal placement strategy does not change effectively. Hence, it is expected that the resulting CRLBs do not differ significantly with respect to discretization once a sufficiently dense grid is employed. Therefore, in the remaining examples, we use the first setup and take 196 points from $\widetilde{\mathcal{R}}$, i.e., $|\widetilde{\mathcal{R}}|=196$.

Fig. 2 presents the average CRLB performance of each algorithm versus $N_{A}$ for two different noise levels: $N_{0}=1$ and $N_{0}=10$. For the same setting, in Fig. 3, the average CRLB performance of each algorithm is plotted versus $1 / N_{0}$ for $N_{A}=3$ and 5 . It is observed that the solution of the relaxed problem is very close to the other two algorithms in both Figs. 2 and 3. Therefore, the optimal solution of the discretized anchor placement problem in (9) is practically achieved in this scenario. Another important observation is that the optimal placement strategy does not change with the noise power, $N_{0}$ since by changing $N_{0}$, we scale all $\lambda_{i}^{(k)}$, s with the same factor. For example, in Fig. 2, the curve corresponding to $N_{0}=10$ is just the scaled version of the curve for $N_{0}=1$.

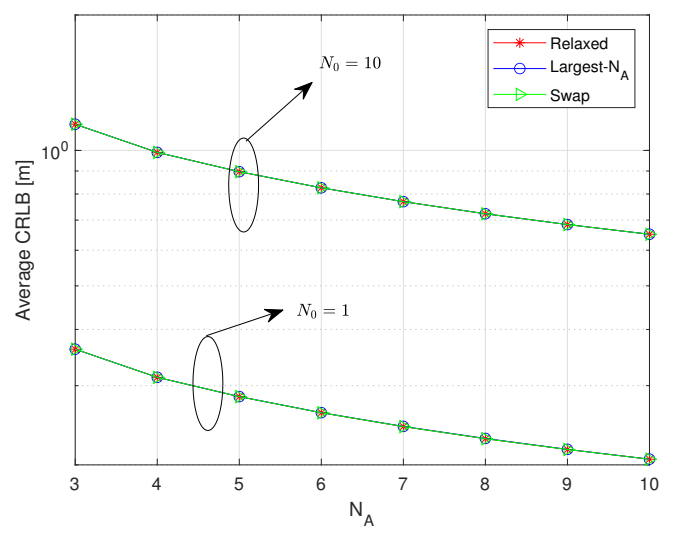

Fig. 2: Average CRLB versus $N_{A}$ for different algorithms when $N_{0}=1$ and 10 .

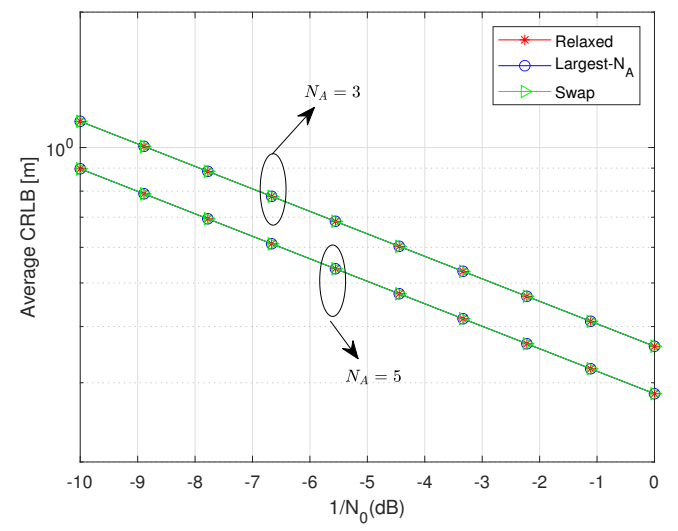

Fig. 3: Average CRLB versus $1 / N_{0}$ for different algorithms when $N_{A}=3$ and 5 .

\section{B. Example of Three Dimensional Wireless Network}

In this part, we consider a wireless localization network as in Fig. 4. In particular, the anchor nodes are placed in $\mathcal{R}=\{[x, y, z] \mid 0 \leq x, y \leq 10, z=10\}$ and for the discretization we take points from $\mathcal{R}$ for each 0.5 meter along both $x$ and $y$ directions such that the number of all points in $\widetilde{\mathcal{R}}$ is equal to 441 . Furthermore, the number of possible target positions is equal to 121 as illustrated in Fig. 4. We use the same simulation parameters as in the two dimensional example except that to realize the shadowing effect, $\lambda_{i}^{(k)}$,s are multiplied by independent log-normal random variables with a mean parameter of -3 and a variance parameter of 3 . For this purpose, we generate a matrix with dimensions $121 \times 441$ by using MATLAB (the seed is equal to 1 ), where the $(i, k)$ th element of this matrix corresponds to the log-normal random variable for the channel between $\mathbf{x}_{i}$ and $\mathbf{y}^{(k)}$ as in [19].

Fig. 5 presents the average CRLB performance of each algorithm versus $N_{A}$ for two different noise levels: $N_{0}=1$ and $N_{0}=10$. We see that when $N_{A}=3$, starting from the solution of the largest- $N_{A}$, via the swap algorithm, we obtain 


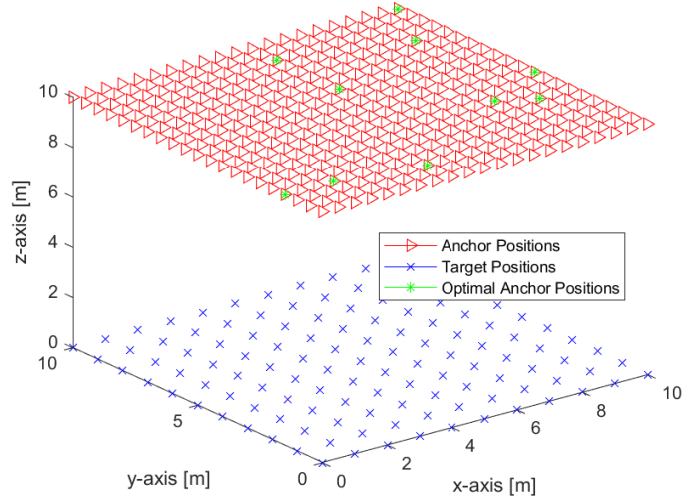

Fig. 4: Illustration of possible locations of target and anchor nodes, together with the optimal anchor locations (obtained from the swap algorithm) when $N_{A}=10$ and $N_{0}=1$.

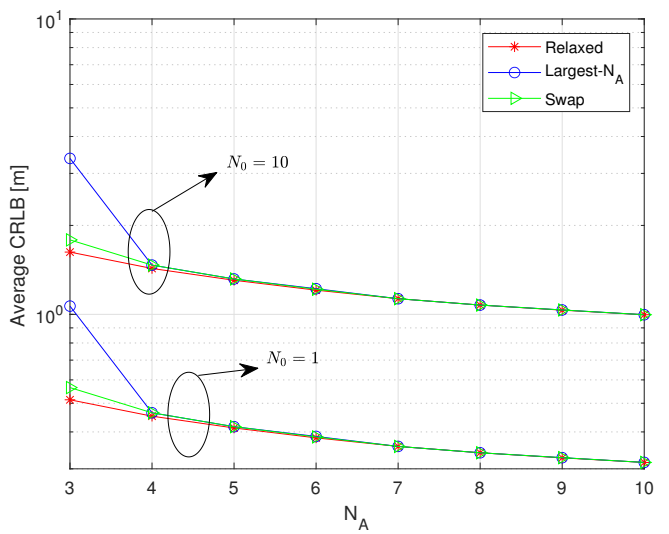

Fig. 5: Average CRLB versus $N_{A}$ for different algorithms when $N_{0}=1$ and 10 .

a solution very close to that of the relaxed algorithm. In other words, in this scenario, we benefit from the swap algorithm. Moreover, in Fig. 5 we again observe that by changing the noise power, we only scale the objective value. For the same setting, in Fig. 6, the average CRLB performance of each algorithm is plotted versus $1 / N_{0}$ for $N_{A}=3$ and 5 . Again the same observations can be made as those for $N_{A}=3$.

Overall, the proposed approach provides an effective solution to the anchor placement problem via discretization and convex relaxation. The performance loss due to discretization can be remedied by using a dense grid, and the suboptimality due to convex relaxation can be mitigated via the swap algorithm, as seen in the numerical examples.

\section{REFERENCES}

[1] R. Zekavat and R. M. Buehrer, Handbook of Position Location: Theory, Practice and Advances, 1st ed. Wiley-IEEE Press, 2011.

[2] M. Z. Win, W. Dai, Y. Shen, G. Chrisikos, and H. Vincent Poor, "Network operation strategies for efficient localization and navigation," Proc. IEEE, vol. 106, no. 7, pp. 1224-1254, July 2018.

[3] H. Zhang, "Two-dimensional optimal sensor placement," IEEE Trans. Syst., Man, Cybern., vol. 25, no. 5, pp. 781-792, 1995.

[4] D. B. Jourdan and N. Roy, "Optimal sensor placement for agent localization," in Proc. IEEE Position, Location, and Navigation Symp. (PLANS), 2006, pp. 128-139.

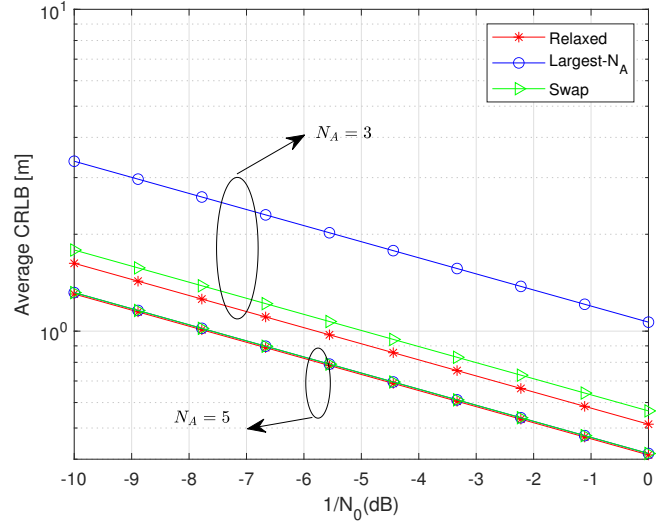

Fig. 6: Average CRLB versus $1 / N_{0}$ for different algorithms when $N_{A}=3$ and 5 .

[5] S. Martínez and F. Bullo, "Optimal sensor placement and motion coordination for target tracking," Automatica, vol. 42, no. 4, pp. 661 $-668,2006$.

[6] Y. Shen and M. Z. Win, "Energy efficient location-aware networks," in Proc. IEEE Int. Conf. on Commun., 2008, pp. 2995-3001.

[7] J. T. Isaacs, D. J. Klein, and J. P. Hespanha, "Optimal sensor placement for time difference of arrival localization," in Proc. 48th IEEE Conf. Decision Control, 2009, pp. 7878-7884.

[8] A. N. Bishop, B. Fidan, B. Anderson, K. Doğançay, and P. N. Pathirana, "Optimality analysis of sensor-target localization geometries," Automatica, vol. 46, no. 3, pp. $479-492,2010$.

[9] J. Y. Zhou, J. Shi, and X. L. Qiu, "Landmark placement for wireless localization in rectangular-shaped industrial facilities," IEEE Trans. Veh. Technol., vol. 59, pp. 3081 - 3090, 082010.

[10] W. Meng, L. Xie, and W. Xiao, "Optimality analysis of sensor-source geometries in heterogeneous sensor networks," IEEE Trans. Wireless Commun., vol. 12, no. 4, pp. 1958-1967, 2013.

[11] Z. Liu, W. Dai, and M. Z. Win, "Node placement for localization networks," in Proc. IEEE Int. Conf. Commun., 2017, pp. 1-6.

[12] S. Xu and K. Doğançay, "Optimal sensor placement for 3-D angle-ofarrival target localization," IEEE Trans. Aerosp. Electron. Syst., vol. 53 , no. 3, pp. 1196-1211, 2017.

[13] W. Bai, R. Iyer, K. Wei, and J. Bilmes, "Algorithms for optimizing the ratio of submodular functions," in Proc. Int. Conf. on Mach. Learn., 2016, p. 2751-2759.

[14] Y. Shen and M. Z. Win, "Fundamental limits of wideband localization Part I: A general framework," IEEE Trans. Inf. Theory, vol. 56, no. 10, pp. 4956-4980, Oct. 2010.

[15] H. V. Poor, An Introduction to Signal Detection and Estimation. New York: Springer-Verlag, 1994

[16] S. Fujishige, Submodular Functions and Optimization, ser. ISSN. Elsevier Science, 2005

[17] S. Boyd and L. Vandenberghe, Convex Optimization. Cambridge University Press, 2004

[18] M. Grant and S. Boyd, "CVX: Matlab software for disciplined convex programming, version 2.1," http://cvxr.com/cvx, Mar. 2014.

[19] C. Ozturk and S. Gezici, "Eavesdropper selection strategies in wireless source localization networks," in Proc. IEEE Int. Conf. Commun. (ICC), 2020, pp. 1-6.

[20] M. F. Keskin, C. Ozturk, S. Bayram, and S. Gezici, "Jamming strategies in wireless source localization systems," IEEE Wireless Commun. Lett., vol. 8, no. 4, pp. 1141-1145, Aug. 2019. 